\title{
Query Optimization in an OODBMS
}

\author{
Christian Rich, Marc H. Scholl \\ Computer Science Department, University of Ulm \\ e-mail: \{rich, scholl\}@informatik.uni-ulm.de
}

\begin{abstract}
It is clearly crucial for the success of object-oriented databases to find efficient implementations that improve on the performance of relational systems, rather than being powerful in terms of modeling and features, but just too slow to be used. This paper describes the mapping of COCOON to DASDBS, a nested relational database kernel system, as an example OODBMS mapping to a complex storage system. We describe 1) choices for physical designs that make use of the complex storage model and 2) the generation of efficient, set-oriented execution plans for object-oriented database queries, using rule-based query optimization techniques. We use hierarchical clustering and embedded (sets of) object references, and show how to explore them for efficient path traversals expressed in queries involving complex objects. Prototypes of both, a physical design tool and a query optimizer have been implemented. Preliminary results show feasibility, and execution time improvements of an order of magnitude.
\end{abstract}

\section{Introduction}

The design of Objectbase Systems, which map object oriented-, semantic- or knowledge representation models to an underlying database kernel system, in order to close the gap between application and database system, are currently considered as candidates for future database architectures. Query optimization in COCOON, an object oriented database project following this kernel architecture approach, will be sketched in this report.

OODBMSs have been designed based on the observation that today's databases supporting simply structured data are quite successful in commercial areas, but future applications need the support for more complex structures, with complex integrity constraints built into the model and with powerful, set-oriented query interfaces. The rationale of the COCOON project is to build an object-oriented model (COCOON), supporting flexibility through powerful structuring primitives, rich semantics, and encapsulation, as well as a powerful query and update language (COOL), on top of a database kernel (DASDBS), which efficiently supports common database operations, such as storage of and access to complex database records (see Figure 1.1). A similar approach is followed e.g. by Härder et al. [6]. 


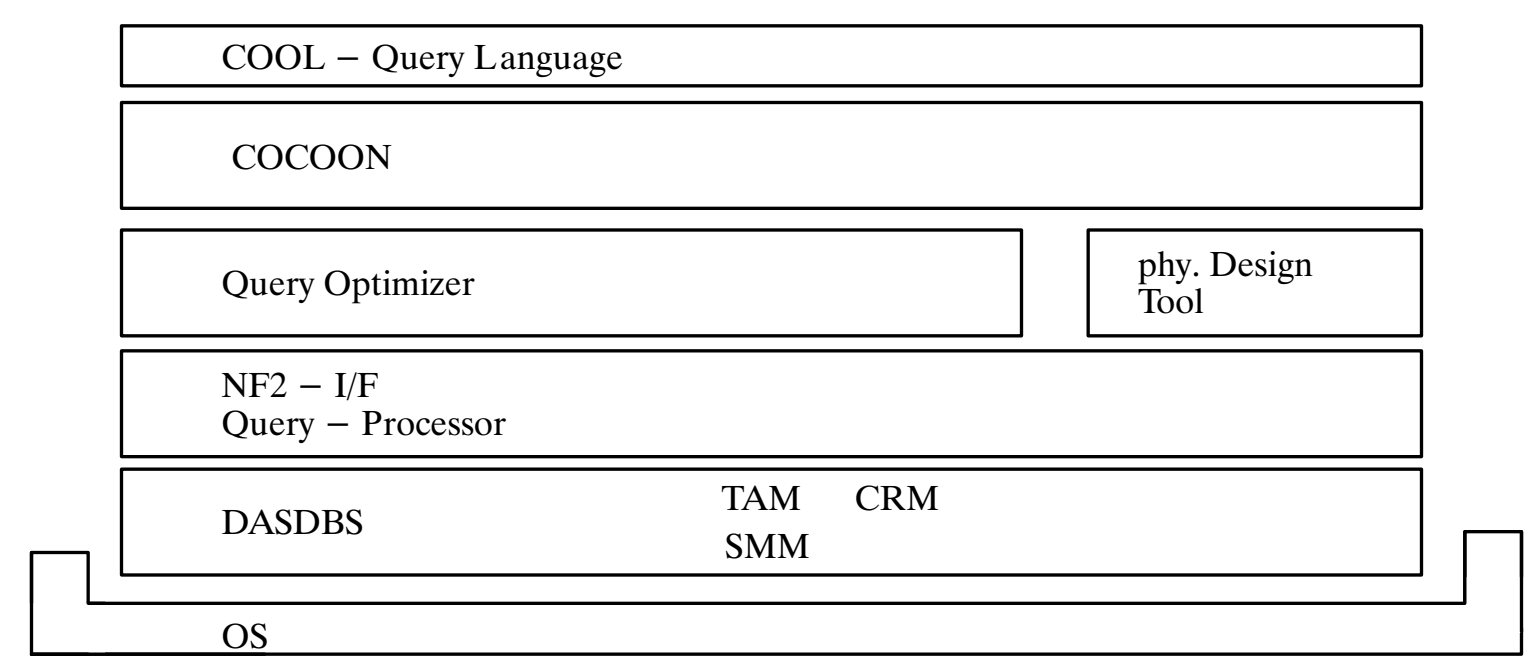

Figure 1.1: The COCOON - DASDBS Architecture

One of the fundamental innovations of the relational approach to databases are the non-procedural query languages, which offers high level, set-oriented database access as well as efficiency through optimizations performed by the DBMS as key advantages. Provided that we accept the requirements of new applications to continue to work with such high-level languages in OODBMSs, we have to: (i) extend "relational-style" languages properly, so as to adopt them to the more powerful models, and (ii) extend "relational-style" query processors to efficiently execute these new query languages.

It is clearly crucial for the success of OODBMSs to find efficient implementations that improve on the performance of relational systems, rather than being powerful in terms of modeling and features, but just too slow to be used. Up to now, no final conclusion can be drawn on how the architecture of an OODBMS should look. It is, however, a common anticipation that relational systems as the underlying storage engine would be to slow. Further, even though the large body of relational query optimization techniques can be used as a starting point for object-oriented query optimization, obviously, to fully exploit the object-oriented paradigm, special optimization techniques are needed.

The flexibility concerning the modeling and processing inherent in the object models and query languages poses a high demand on the implementation. On one hand, even more so than in traditional databases, the conceptual structure of the database is hardly ever also an efficient, internal one. Rather, data representing the conceptual objects may be structured completely different for performance reasons. Therefore, the mapping of objects of the model into the structures of the DBMS kernel system, has to be considered with great care. The most favorable internal representation is dependent on the type and frequency of expected queries, that is, the transaction load faced. On the other hand, in order to support efficient application execution, all issues related with query evaluation and optimization as well as general considerations about the mapping of functionality onto the kernel system have to receive special attention. 
We used DASDBS, our prototype database kernel system, which supports flexible structures with operators that go beyond relational queries, as the target platform for the research described below. A query optimization methodology is specified, and a first query optimizer has been implemented accordingly. It was one of the main objectives of the COCOON project to reach some (at least preliminary) conclusions on the feasibility and performance trade-offs of this approach. DASDBS was chosen as the storage system, because of its support for complex storage structures. First of all, the support of nested relations allows for the storage of hierarchically clustered data. That is, we have hierarchical access structures with an arbitrary level of nesting, as well as the opportunity to define nested join indices. This can be very useful to store COCOON objects and the "relationships" between them. Second, the DASDBS interface offers powerful data retrieval and manipulation operations. The system has a set-oriented, algebraic interface with efficient operations on complex objects. All operations performed in the kernel are executed in a single scan through the data [22,26]. Operations that are not single scan processible are performed outside the kernel, in the higher-level query processor. The query processor's ability of processing on complex nested structures, further improves processing, like for reassembling complex object structures [25].

The overall architecture of the COCOON implementation on top of the DASDBS kernel system is shown in Figure 1.1. The two aspects of this architecture, structure mapping and operations mapping, are realized in the physical design tool and the query optimizer, respectively. Physical design uses the COCOON schema, statistics about cardinalities and distribution, and a description of a transaction load to propose a good internal storage structure expressed in terms of nested DASDBS relations. At transaction processing time, the optimizer has to translate COOL operations down to operations on these physical $\mathrm{NF}^{2}$ structures. The execution plans generated consist of physical $\mathrm{NF}^{2}$-algebra operators, some of which, such as joins, are implemented in the highlevel query processor, others are DASDBS kernel calls.

The paper is structured as follows: Section 2 gives a short description of COCOON and introduces a running example, before we describe physical design choices in Section 3. In Section 4 we present the translation of queries onto the physical level, and the optimization of query execution plans. Section 5 compares with related work, before we conclude in Section 6.

\section{COCOON}

Essentially, the COCOON model as described in $[31,32]$ is an object-function-model. Its constituents are objects, functions, types and classes. COCOON offers a variety of structuring capabilities to model complex objects. Class and type hierarchies (i.e. classification and generalization), the abstraction concepts of aggregation and association, and derived methods offer a flexible modeling framework. The query language, COOL, offers object-preserving as well as object-generating generic query operators plus generic update operators. The key objective in the design of COOL was its set-oriented, descriptive characteristics, similar to a relational algebra. COCOON is a core object model, basically we have objects (concrete and abstract) and one 
type constructor, namely set. Other features can be added later due to the orthogonality of the language.

Objects are instances of abstract data types (ADTs). They can be manipulated only by means of their interface, a set of functions. Data are instances of concrete types (such as numbers, strings) and constructed types (such as sets). The distinction from objects is similar to [2].

Functions are described by a name and signature (i.e., domain and range types). Functions can be single - or set-valued, they are the interface operations of types. A useful feature is the capability of defining inverses of functions. This integrity constraint is enforced by the system during updates.

Types are described by their name and the set of functions that are applicable to their instances. Types are arranged in a subtype hierarchy, where subtypes inherit functions from their supertypes. Objects can be instances of more than one type at the same time ("multiple instantiation")

Classes are typed collections of objects (sometimes called "type extends"). Classes are arranged in a subclass hierarchy that is exactly the set inclusion between the sets of objects they represent. Objects are "members of" classes, possibly more than one at a time ("multiple class membership"). Particularly, superclasses contain all members of their subclasses.

As an example, we will use an architectural design application throughout this paper. The COCOON graphical representation is given in Figure 2.1 In this application we are mainly dealing

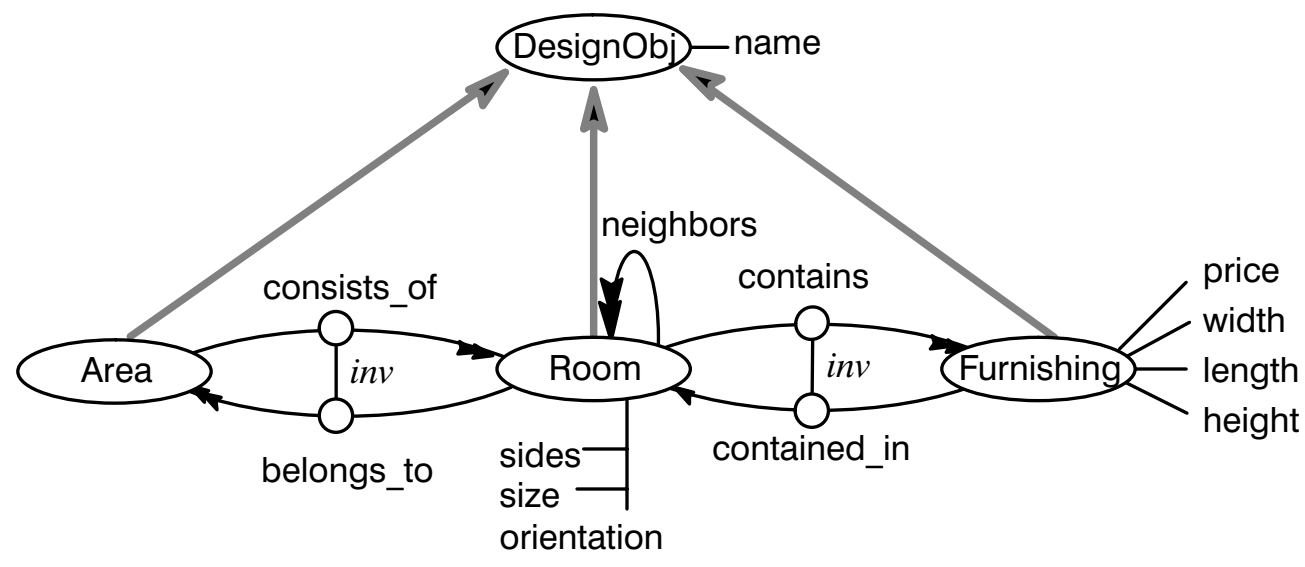

Figure 2.1: Our example DB-World

with rooms, furnishings and areas. All of them are design-objects. Rooms are described by their name, orientation, position, size, etc. Between certain rooms there is a neighborhood-relationship. Each room contains a set of furnishings, which are described by their name, price, width, length, height, etc. Each room belongs to some areas, and each area consist of some rooms. That is, the room 'Office1' could be part of the area 'working - area', and may contain furnishings like 'Wooden Desk' and 'Computer'. A COOL query asking for offices, having no 'computer' is given below:

$$
\begin{aligned}
& Q:=\text { select }[\text { name }=\text { "Office" } \\
& \text { and select [name }=\text { "Computer"] (contains })=\{\}](\text { Room }) ;
\end{aligned}
$$




\section{Physical Database Design}

In this section we discuss the mapping of COCOON schemas to nested relations (DASDBS) [30]. That is, given a COCOON database schema, what are the alternatives for the internal DB layouts, and further, given a transaction load, which internal DB layout results in the least overall cost of transaction execution. This optimization task is performed by the physical design tool.

Because COCOON (like almost all OODBMS) offers a variety of structuring capabilities to model complex objects, it is not at all trivial to find good, that is, efficient, storage structures that support the variety of operations on objects reasonably well. Objects may be hierarchically composed of subobjects, several objects may share common subobjects, objects may appear as (attribute) values of other objects, different objects can be related to each other by functions, methods, or relationships. Type (or class) hierarchies introduce another dimension of object interrelation: an object of one class also "appears" in all its superclasses; again, with multiple inheritance, this need not be a strict hierarchical inclusion. Computed values (attributes, methods) may be used to derive, rather than store, data that are associated with objects.

\subsection{Alternatives for Physical DB Design}

In order to explain the alternatives for mapping $\mathrm{COCOON}$ database schemas to nested relations at the physical level, we proceed by stepping through the basic concepts of the COCOON object model, and showing the implementation choices. Since the choices for each of the concepts combine orthogonally, this spans a large decision space that is investigated by the physical database design tool (next section).

\section{Implementing Objects}

According to the object-function paradigm of COCOON, an object itself is sufficiently implemented by a unique identifier (OID), that is generated by the system. All data related to an object in one way or the other will refer to this identifier (see below). We denote for each object type $A$, attributes of internal relations containing the OID of objects of type $A$ by $A I D$.

\section{Implementing Functions}

In COCOON, functions are the basic way of associating information (data values or other objects) to objects. In principle, we can think of each function being implemented as a binary relation, with one attribute for the argument OID and the other for the result value (data item or OID). In case of set-valued functions the second attribute will actually be a subrelation of unary subtuples, containing one result (OID or data value) each. So, in principle, each single-valued function as well as each multi-valued function would be implemented in a binary relation. For example, in our architectural design application, the functions size and furnishings of Rooms would result in two relations, Room_size(RID, size) and Room_furnishings(RID, furnishings(FID)) respectively. Obviously, there are some choices. The decision space as far as function implementations are concerned includes the following alternatives in our current approach:

Bundled vs. Decoupled: Each function defined on a given domain object type might either be stored in a separate (binary) relation as shown above: the decoupled mode. Alternatively, we 
can bundle functions together with the relation implementing the type.

In the example above, the bundled implementation of Room size and Room furnishings, would yield the following type table : Room(RID, size, furnishings(FID)).

Logical vs. Physical Reference: A function returning a (set of) object(s), not (a) data value(s), can be implemented by storing just OIDs (logical reference) of result objects or by including a TID (physical reference) as well. Continuing on the above example (bundled), inclusion of physical references for the neighbors function, would result in:Room(RID, size, furnishings(FID, @F)). Notice the naming convention: (physical) reference attributes have @ as a prefix.

Oneway vs. Bothway References: A function can be implemented by a forward reference only (oneway), or it can be implemented with backpointers (bothway). For example, in case the contained_in function of furnishings would not be present in the conceptual schema, we could nevertheless decide to implement it, to have the contains function of Rooms supported with backpointers as well.

Reference vs. Materialized: Functions returning (possibly sets of) objects, not data values, can be implemented by the various forms of references discussed up to now. Alternatively, however, we can directly materialize the object-tuple(s) representing the result object(s) within the object-tuple representing the argument object. This is a way of achieving physical neighborhood (clustering). In our example, the decision to materialize the furnishings function of Room objects would generate a nested type table that contains the type table for furnishings as a subrelation: Room(RID, size,... furnishings(FID, name, ...)). Obviously, we need no backward references in this case. Furthermore, this alternative is free of redundancy only if the materialized function is $1: n$, that is, it's inverse is single-valued.

Computed vs. Materialized: Finally, an additional option is to materialize derived (computed) functions. Assuming that some function can be computed, we could nonetheless decide to internally materialize it, if retrieval dominates updates to the underlying base information significantly. The more retrieval dominates updates, and the more costly the computation is, the more likely is the case that materialization pays off. For example, the size function of Rooms is derived from the actual geometry of the Room. But computing the size incurs quite some effort and if object shapes rarely change, materializing the size function clearly is a good strategy (see also [10]).

\section{Implementing Types, Classes, and Inheritance}

The COCOON model separates between types and classes, this results in having two inheritance hierarchies: one between types (organizing structural, function inheritance), and one between classes (organizing set inclusion). Since classes are always bound to a particular (member-) type, physical design for types is the larger grain approach, whereas design for individual classes would be the finer grain approach (remember, there may be more than one class per type). Currently, we do the physical design on a type basis, that is, all objects of a given type are physically represented in the same way (even if they belong to several classes). Classes are implemented as views 
over their underlying type table. This results in the following choices w.r.t. types, classes, and inheritance:

Types: Each object type is mapped to a type table with at least one attribute, containing the OID. Additional attributes are present in case of any bundled functions and/or materializations of object functions. The type table $T$ may itself be a subrelation of some other table, if type $T$ was materialized w.r.t. a function returning $T$-objects.

Classes: Each class $C$ is implemented as a view over its underlying type table. If the class is defined by a predicate ("all" - classes and views in COCOON), this predicate is used as the selection condition. If the class is defined to include manually added member objects ("some"-classes in COCOON), the underlying type table is extended by a Boolean attribute $C$ that is set to true, if the object is a member of this class $C$.

Inheritance: Subtyping is implemented by having one type table per subtype. Three possibilities are considered:

- an object - tuple is included in each supertype's table. In case there are any bundled or materialized functions, these are not repeated in the subtypes' tables. In this case, object-tuples in subtype tables might optionally include physical references to supertype tuples.

- an object-tuple is included only in one type's table, that of the most specific subtype. In case of any bundled or materialized functions in supertypes, these are also included in the subtype's table.

- an object-tuple is included in each supertype's table. In case of any bundled or materialized functions in supertypes, these are also included in the subtype's table.

\subsection{The Default Physical Design}

In order to have a starting point for the implementation of COCOON on top of DASDBS, we have identified a default physical design that includes the following choice of implementation strategies:

Functions: All functions are bundled with their type table. Object-valued functions are implemented as references, with physical references and backpointers. Multi-valued functions become subrelations.

Inheritance: Objects are present in all supertype tables, inherited functions are repeated in subtype tables. No backpointers to supertype tuples are included.

The default physical design for our architectural example world, given in the beginning (Figure 2.1), is shown in Figure 3.1.Further, we show an alternative physical design, where the object valued function furnishing is materialized, in Figure 3.2. Both designs will be used later on, when describing the query optimization task.

\subsection{Physical Design Tool}

In order to exploit the options given above, we have a first prototype implementation of a "physical DB design expert system" (considering some of the above alternatives) running. The system 


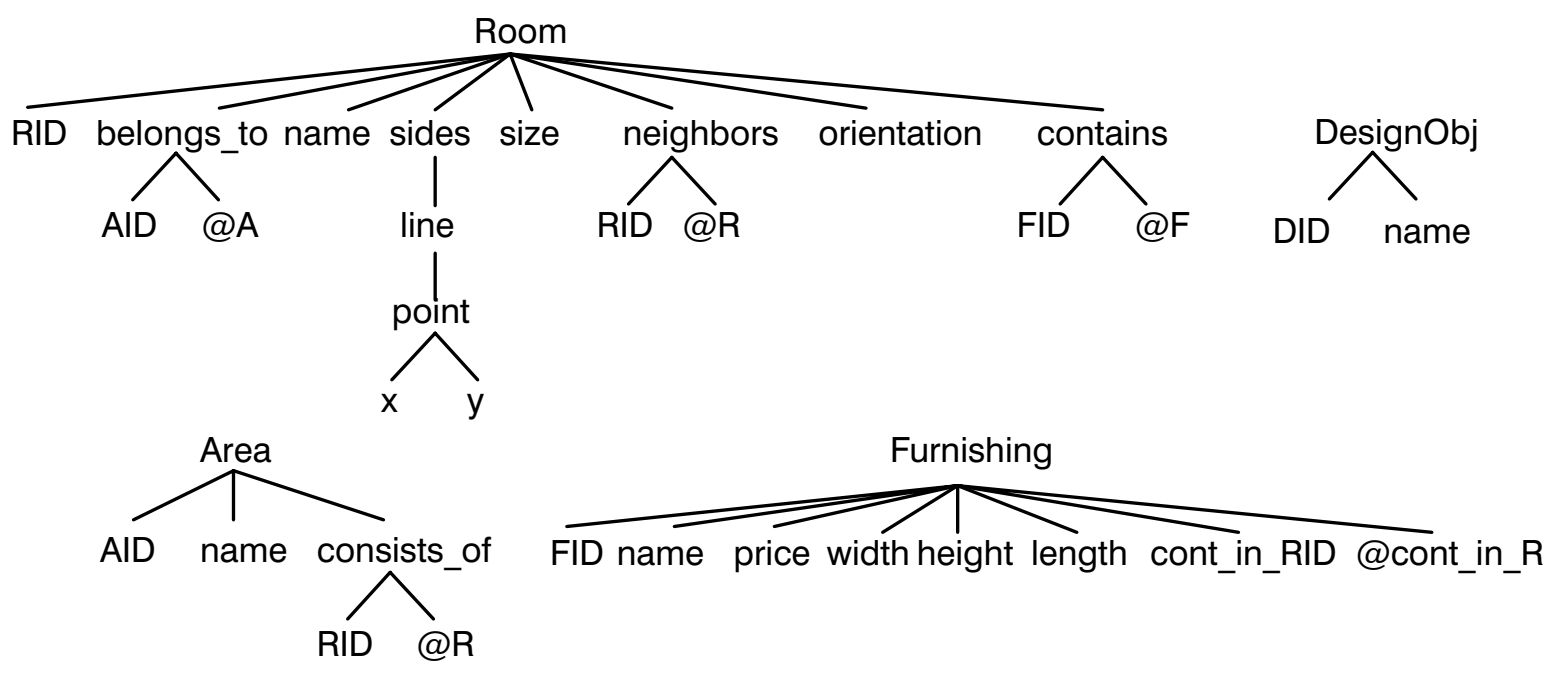

Figure 3.1: Physical Design I of our Example DB-World

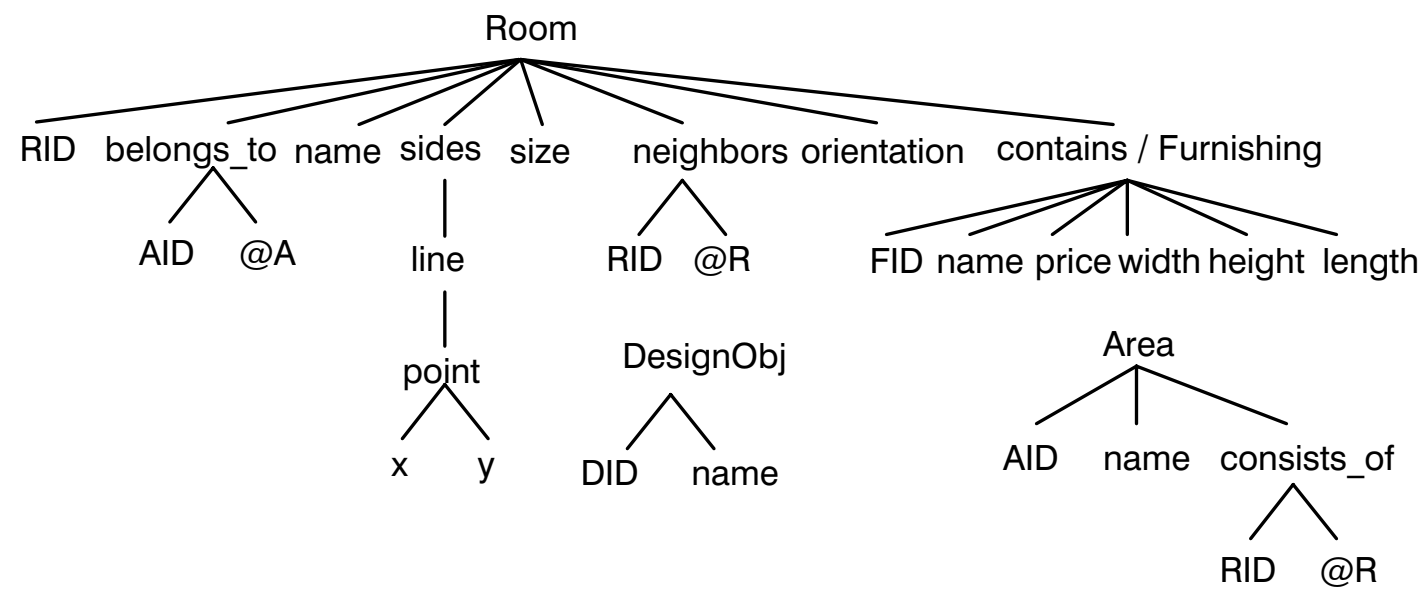

Figure 3.2: Physical Design II of our Example DB-World

takes as input a COOL schema, a description of the anticipated (or observed) transaction load, and information on the cardinality and size of the DB objects. The output is a (set of) proposed physical designs for use with DASDBS, together with cost estimates for the transaction mix obtained from a cost model. The system is implemented in Prolog, and will be extended to consider a wider choice of storage schemas, especially those including access paths and more general forms of redundancy in the future.

\section{Query Optimization}

In this section, we discuss the transformation and optimization of queries that are given to the system in terms of the COCOON database schema. It is the task of the query optimizer to map these COOL queries down to the physical level by: (i) transforming them to the nested relational model and algebra as available at the DASDBS kernel interface, and (ii) select a good (if not the best) execution strategy.

Because COCOON's query language, COOL, is pretty similar to a nested relational algebra, a straightforward transformation from COOL expressions down to a nested relational algebra expression against any fixed implementation on the internal level (e.g., the default physical design) 
is rather easily done. Complications arise from the fact that the mapping of data structures is very flexible, and that, depending on the chosen design, operations have to be optimized substantially.

We have investigated two competitive approaches to query transformation and optimization. The first one is a purely algebraic one, comparable to what we did with the relational to nested relational mapping [27]: COCOON classes would be defined as 'views' over the stored nested relations, COOL queries would be transformed to the nested relational level by 'view substitution', and finally, algebraic transformations within the nested relational algebra could be applied, so as to eliminate redundant subexpressions. Quite a few redundant joins would have to be removed in case of materialized functions (hierarchical clustering). This has exactly been the problem addressed in [29].

Example 4.1: Given the COOL query

$$
\begin{aligned}
Q_{1}:=\text { extract [ } & \text { name, orientation, size, } \\
& \text { extract[ name ] ( neighbors ), } \\
& \text { extract [ name, price, width, length ] (contains) } \\
& (\text { Room ); }
\end{aligned}
$$

and assuming the default physical design (Figure 3.1) in the transformation of this query to the $\mathrm{NF}^{2}$ level, this results in the query

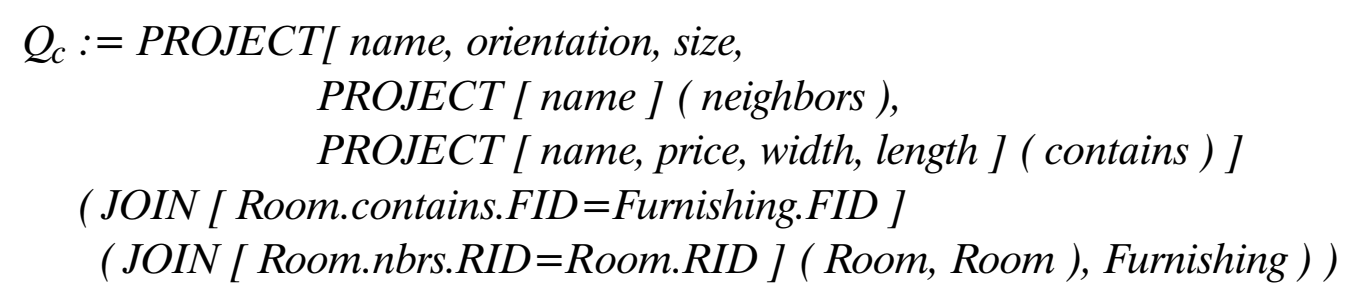

However, if the internal physical design is the one given in Figure 3.2, that is the function contains is materialized, one join operation can be removed by optimization, resulting in the query $\mathrm{Q}_{\mathrm{i}}$. Notice, that further optimization could be performed, which is not shown here.

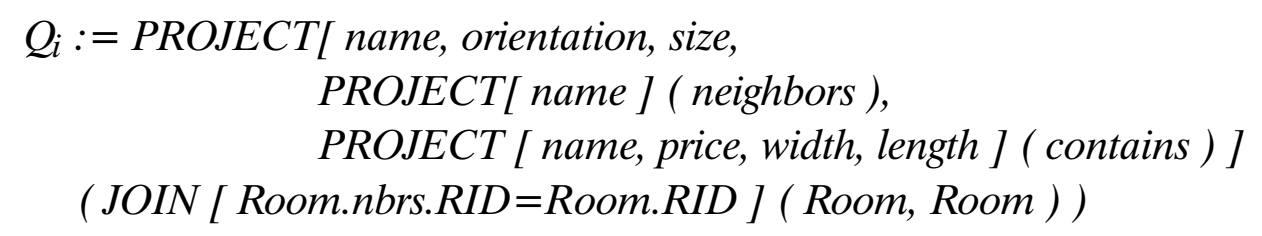

The second approach directly uses the information about the physical database schema in the transformation of a given COOL query into a nested relational algebra representation. No redundant joins are created, that would have to be removed in the following optimization phase. Thus we have a more direct transformation. In Example 4.1 above, this would result in transforming query $\mathrm{Q}_{1}$ directly onto the physical schema, that is, into query $\mathrm{Q}_{\mathrm{i}}$.

We have chosen this second approach in our current implementation. The optimization phase following the transformation chooses the specific execution strategy, e.g. the ordering of operators, as well as the best implementation strategies. For example, whether a nested loop or a sort merge join is selected. This leads to the architecture of our query optimization process, given in Figure 4.1. 


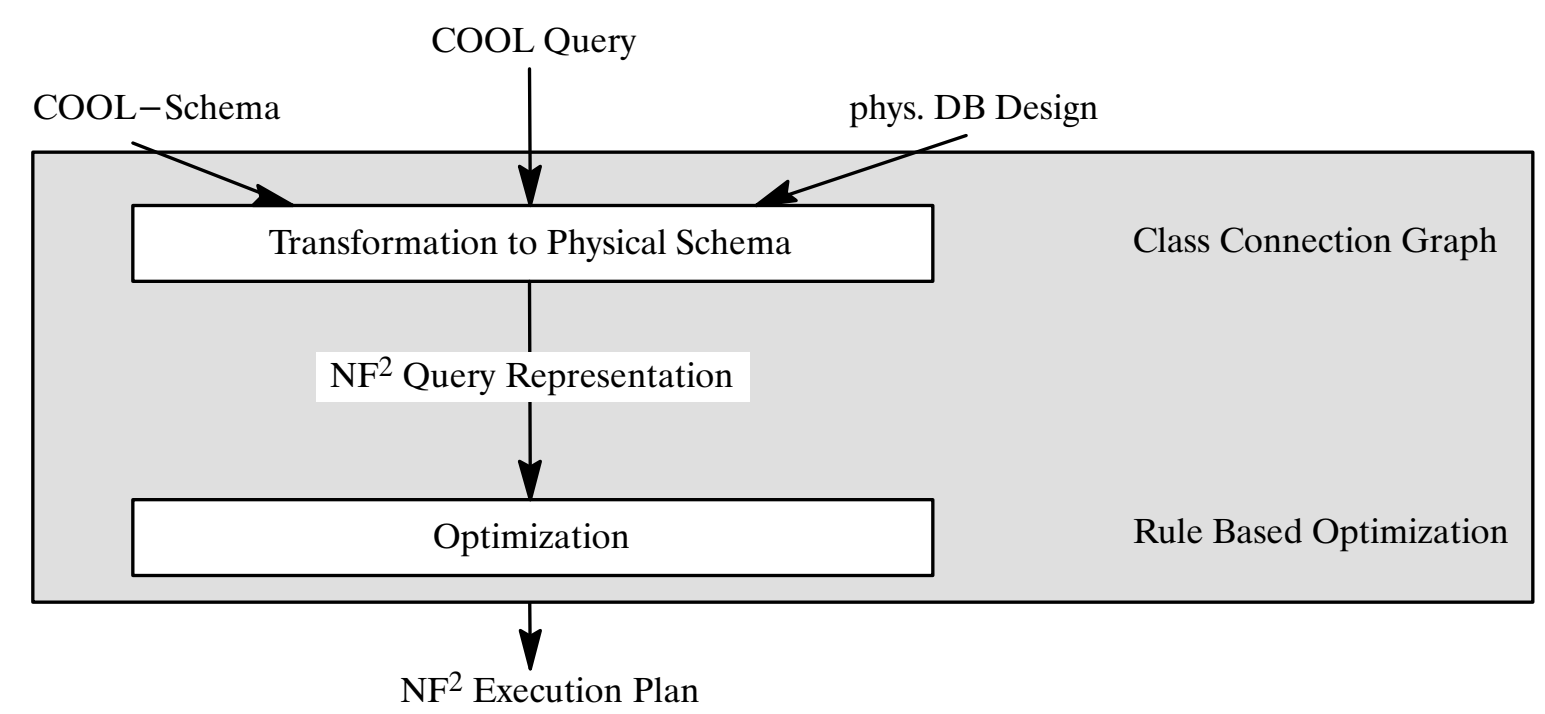

Figure 4.1: Optimizer Architecture

In the first step, the transformation task, we use a "Class Connection Graph". We will elaborate more on that in the following. The second step, that is, the optimization phase, we use a rulebased algebraic query optimizer, generated with the EXODUS Optimizer Generator [8].

\subsection{Transforming COOL-Queries onto the Physical Schema}

The input to the optimizer is a COOL-query expressed on the conceptual schema, while the transformations apply to execution plans, i.e., on the physical schema. The first step is to do the translation to an algebraic query representation on the physical schema. To do this, we use a Class Connection Graph similar to the one proposed in [17].

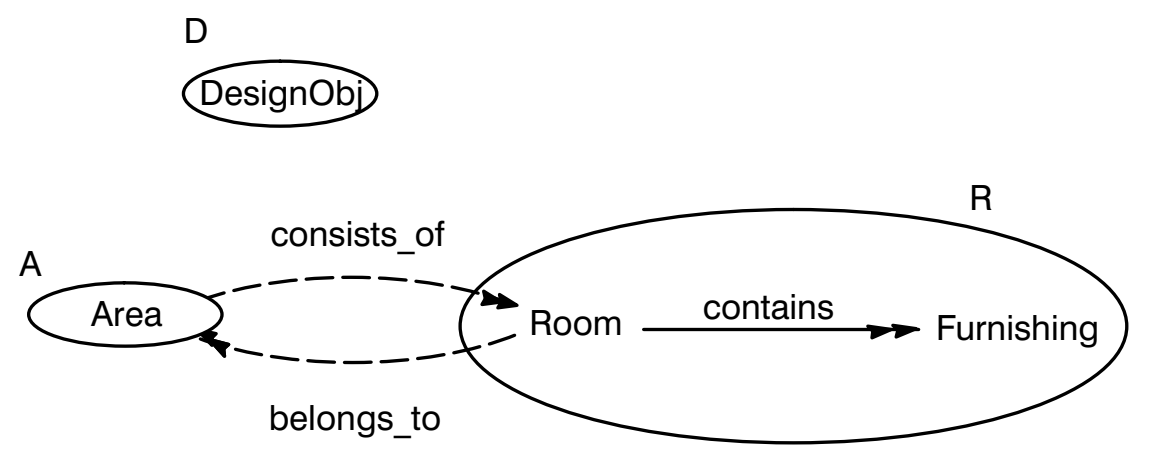

Figure 4.2 : A sample Physical Schema Graph

After the input query is parsed, the optimizer scans at the same time the query graph (a graph representing the given query) and a physical schema graph. From these, the class connection graph is constructed. A possible physical schema graph is given in Figure 4.2. This schema graph corresponds to our physical design, shown earlier in Figure 3.2. The nodes are files, which may implement

- a single class extension (e.g. A implements Areas and D implements DesignObjects) 
- several class extensions (e.g. R implementing Room and Furnishings)

- a part of a class extension, when a class extension is vertically fragmented.

The arcs denote the kind of function implementation. Solid arcs denote materialized functions (e.g. contains). Dashed arcs are functions stored as references to subobjects inside the instance of the owner object.

Now let us look at the transformation of a sample query. Suppose we have a physical schema graph as shown in Figure 4.2 and the following query:

Query $Q 2$ :

extract [ name, orientation, size,

extract [ name ] ( neighbors),

extract [ name, price ] ( select [ name = "computer"] ( contains $))$

]

$(\operatorname{RoomC})$

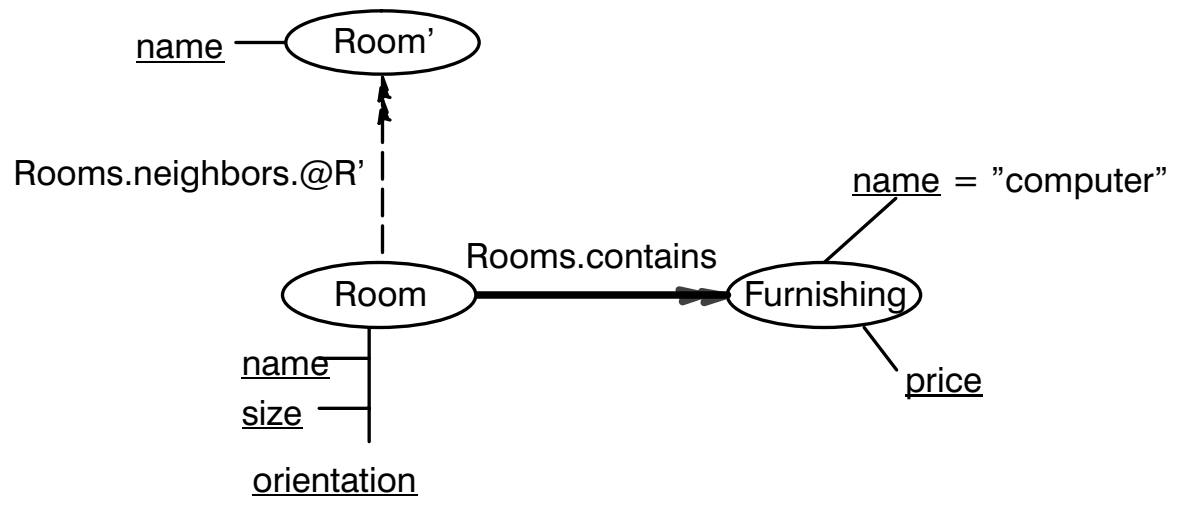

Figure 4.3: A sample Class Connection Graph

Figure 4.3 shows the Class Connection Graph constructed for that query. The nodes of the class connection graph represent classes which are affected by the given query. The edges are the functions involved in the query, connecting classes via implicit or explicit joins. There are three different kinds of edges, depending on the physical representation of these functions, i.e. did we store logical OIDs, physical references, or is this object function materialized. In our example, the contains function of Room objects is materialized, and the neighbors function is supported by pointers. The use of the neighbors function results in a second occurrence of class Room, here denoted as Room'. In case a class extension would be stored vertically fragmented, this would result in having a class for each fragment, all of them connected with corresponding arcs. Further, we see the selection predicates (name $=$ "computer") and the printable attributes, which shall be displayed (name, size, and orientation of Rooms).

A class connection graph represents a given query, without any hints on execution orders, that is, all possible execution plans can be generated from the class connection graph. For example, we have the choice to perform forward or backward traversals, or to start in the middle of a path, as well as to interleave other operations with the traversal (cf. [21]). 


\subsection{Optimization of Query Execution Plans}

In this section we describe the model of execution offered by the base system (DASDBS kernel and query processor) and how query execution plans are represented. Then, we describe the opportunities for optimization, give some example transformation rules defining the search space, and show how to explore it. Further, we will give some example queries and demonstrate how they are optimized by our system.

\subsubsection{Model of Execution}

The DASDBS kernel interface is set-oriented, with efficient operations on complex objects. Due to the support of nested relations, complex objects may be hierarchically clustered. However, there will be the need to reassemble objects, which are not clustered in one nested relation, as well as to perform operations on these reassembled objects. These tasks are performed in the query processor (see Figure 1.1). The resulting model of execution is as follows: the run-time system consists of a limited set of procedures, the physical algebra operators. Each procedure transforms a data stream according to an argument which was derived from the original query. For example a selection operator is removing tuples of a data stream, not satisfying the corresponding predicate given in the query. Complex queries result in nesting these procedures, i.e. the output of one procedure will be the input of another one. The transfer of data between these procedures is done by streams [7], to avoid unnecessary writing of intermediate results. All operators are performed within a single operating system process, to prevent operating system scheduling and inter-process communication, as it is much more expensive than procedure calls.

Our approach to describe the nesting of base system procedures, is to use processing trees. A processing tree represents the execution plan for a given query. Two possible processing trees are given in Figure 4.4. The leaf nodes, which represent complex operations on one DASDBS relation, are performed (in a set-oriented way) by the DASDBS kernel. Internal nodes are performed in the COOL-specific query processor (in a streaming mode). Again, it is obvious, that the initial tree which is derived from the original query, will probably not be the optimal to execute. It is the optimizers task to find the cheapest equivalent execution plan, which then will be executed.
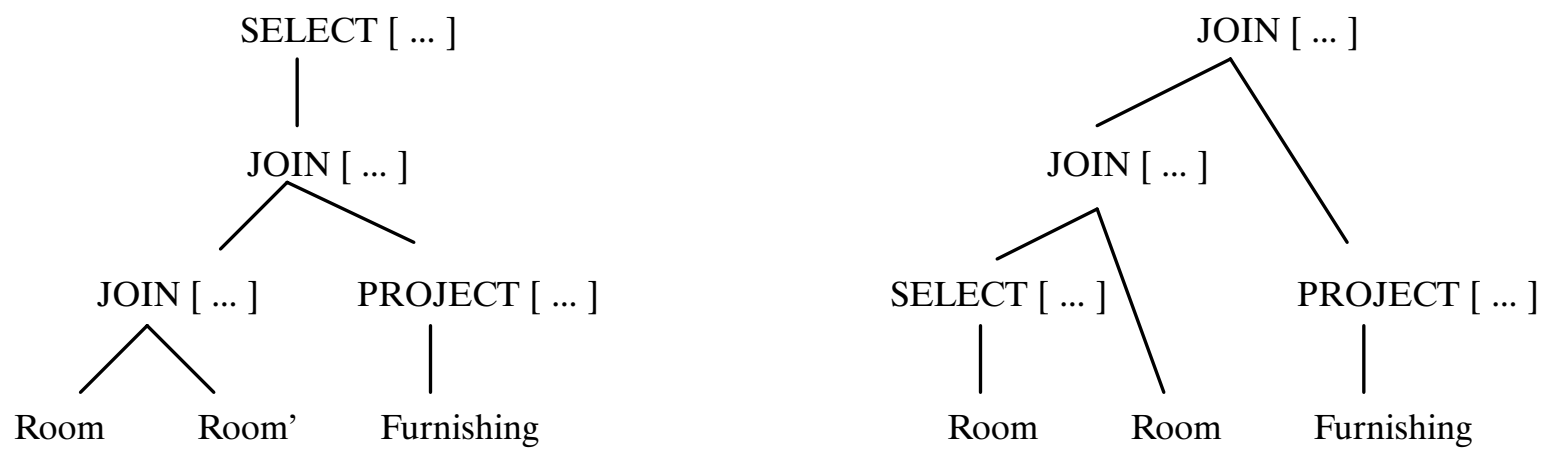

Figure 4.4: Two equivalent Processing Trees 


\subsubsection{Which Potential for Optimization do we have?}

Given a query, obviously there are many equivalent processing trees, that is, alternatives to execute the query. These alternatives result from a number of open choices, some of which are listed in the following.

Join Ordering. A sequence of join operations is freely reorderable, this results in many alternatives. In order to reduce the number of orderings to consider, one may exclude the ones resulting in Cartesian products, or restrict the join orderings to the ones resulting in linear join trees, instead of considering all possible, bushy ones (this is the well-known problem studied, for example, in [33, 20, 39, 38].

The reason for join operations to occur is the following: First, reassembling objects from several relations requires a join operation for each partition. Object partitioning is introduced for example, in the mapping of inheritance hierarchies. Second, each application of an object valued function (which is not materialized) results in a corresponding join. Reordering these joins corresponds to changes to the order of function application, that is whether we do forward or backward traversals, or starting in the middle of a path query [3]. Notice, that these implicit joins may be supported by link fields (see Section 3.1), and therefore enable efficient pointer based join algorithms [36].

Pushing Selections. Selections may be performed at various times. For example, one could perform a given selection before or after applying an object valued function (move selection into join, or vice versa). Additionally, selections with conjunctively combined selection predicates may be split into two selections (or vice versa), and the order of selections, as well as the order of the terms in selection predicates, may be changed.

Pushing Projections. Projections, in the same way as selections, may be performed at various times. In addition, the order of applying projections and selections may be changed.

Method Selection. For each logical operation there may be several methods, that is physical implementations. For example, a join operation may be performed by nested loops join, hash join, or sort merge join. In addition, for implicit joins which are supported by link fields, pointer based join versions may be selected. Further, selections may be performed by using indexes, or by performing a relation scan, followed by predicate testing.

Index Selection. If a selection is supported by multiple indices, one has the opportunity to use all, some, or just one of the available indices.

Almost all of the choices given above, can be combined orthogonally, such that the number of equivalent processing trees grows extremely fast with increasing query complexity.

\subsubsection{How to find the Optimal Execution Plan}

A query optimizer should find a good (if not the best) execution plan as fast as possible, i.e. without considering as many as possible alternative execution plans, which will (at least with high probability) not lead to the optimal one. As indicated in the context of extensible DBMS, a rulebased approach to query optimization benefits from two circumstances: first, this allows rather 
flexible extensions to the underlying equivalences or transformation rules, and second, a combination of equivalence-based transformations (i.e., algebraic optimization) with cost-based transformation rules can easily be obtained in this framework. Due to the fact that a rule based query optimizer is one of the most intricate subsystems of a database system, it is desirable not to start an implementation from scratch. Therefore, we decided to use the EXODUS optimizer generator [8] for the implementation of the COOL optimizer. Without going into detail, a cost model to estimate the quality of execution plans, as well as rules to describe possible transformations have to be defined by the implementor of the optimizer, the search strategy is provided by EXODUS.

The cost functions for methods are fairly standard, similar to the ones of System R [33]. They had to be adapted to the nested relational model. These functions are based on information about the stored data, like tuple and page cardinalities for each relation, and the number of distinct values for each attribute. Further improvements, like including information about data distribution, as well as taking indexes into account, are planned for the future.

To specify rules describing possible transformations of processing trees, that is, of nested relational algebra expressions, we utilized transformation rules known from the (flat) relational model and the (complex) rules defined for the nested model in [29, 28] as a starting point. Roughly, $1 \mathrm{NF}$ rules are applicable in the $\mathrm{NF}^{2}$ model on relations and on the subrelations, having additional constraints and more complex argument transfer functions. $\mathrm{NF}^{2}$ rules handle complex operations e.g. nest, unnest and complex select-project-join queries. To handle object-oriented paradigms, such as set valued object functions, inverse functions, or link supported functions, special rules are necessary. For example object reassembly, involving set-valued object functions results in (multiple) joins. Here, the classical join enumeration problem does no longer apply, we have to solve a hierarchical join scheduling problem. The result of a join operation is a hierarchical view on the same set of objects, having an additional nesting. Object reassembly results in one additional nesting for each set valued function. Therefore, changing the join order according to the commutativity and associativity rule, as known from the relational model, may not be correct anymore. A survey of transformation and implementation rules used, can be found in [24].

The EXODUS optimizer generator was designed with the intention to be extendible, that is, it should support query optimization without making restrictive assumptions about the data model, the query language, or the run time system. So using the EXODUS optimizer is in principle quite easy. Looking deeper into it, it turned out that due to the enhanced complexity of the rule set, in addition with more sophisticated constraints and "argument transfer functions", a considerable amount of additional work has to be done by the optimizer implementor.

Example rule: The rule how two project operations can be combined into one project operation, can be formulated in the relational model in the following way:
$\pi[A](\pi[B](E)) \equiv \pi[A](E)$
(Obviously, $\mathrm{A} \subseteq \mathrm{B}$ has to be fulfilled, to have a correct expression on the left side.) 
This rule leads to several rules in the $\mathrm{NF}^{2}$ model, each having an increased complexity. One resulting rule is given below:

$$
\pi\left[\ldots \sigma_{F_{2}}(A) \ldots\right] \pi\left[\ldots \sigma_{F_{1}}(A) \ldots\right](E) \equiv \pi\left[\ldots \sigma_{F_{2}} \sigma_{F_{1}}(A) \ldots\right]
$$

Using the notation enforced by EXODUS, this rule is not expressible, rather it has to be formulated in the following way (actually, in the same way as the relational rule):

$$
\pi 1(\pi 2(E)) \equiv \pi 1(E)
$$

But this rule is rather incomplete, the missing parts have to be supplied in so-called support functions, that is, the condition code (to check whether the rule is applicable) and argument transfer functions.

\subsection{Example Transformation and Optimization}

In the following we will give one more example COOL query, and demonstrate how the query is mapped to an execution plan for the query processor, and where performance gains are achieved.

A query asking for offices without a computer, is formulated in COOL in the following way.

$$
\begin{aligned}
& Q_{3}:=\text { select }[\text { name }=\text { "Office" } \\
& \quad \text { and select }[\text { name }=\text { "Computer"] }(\text { contains })=\{\}] \\
& (\text { Room }) ;
\end{aligned}
$$

Assuming the physical design I (Figure 3.1), the translation to the physical schema results in a processing tree as given below.

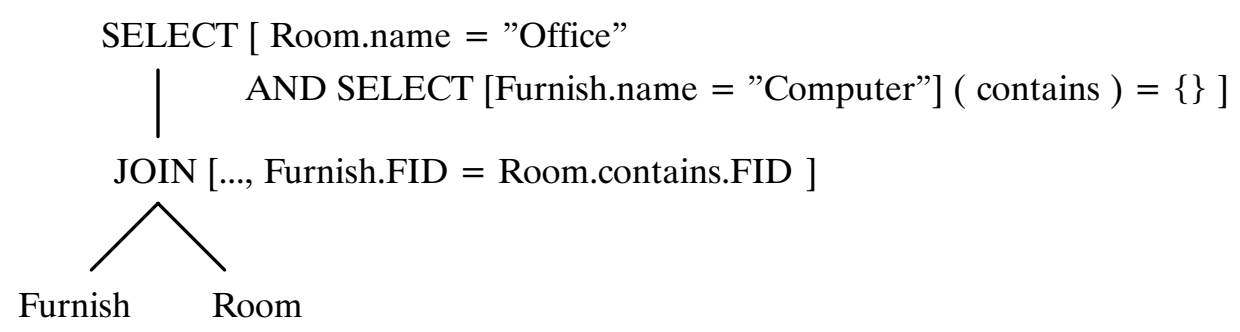

Note that due to the use of the contains function (which is not materialized), a join operation is inserted. Because there is no partitioning or subtyping, no additional joins are necessary in this example. The '...' after the keyword JOIN indicate, that (up to now) no specific join method has been selected. After optimization, the resulting execution plan looks like:

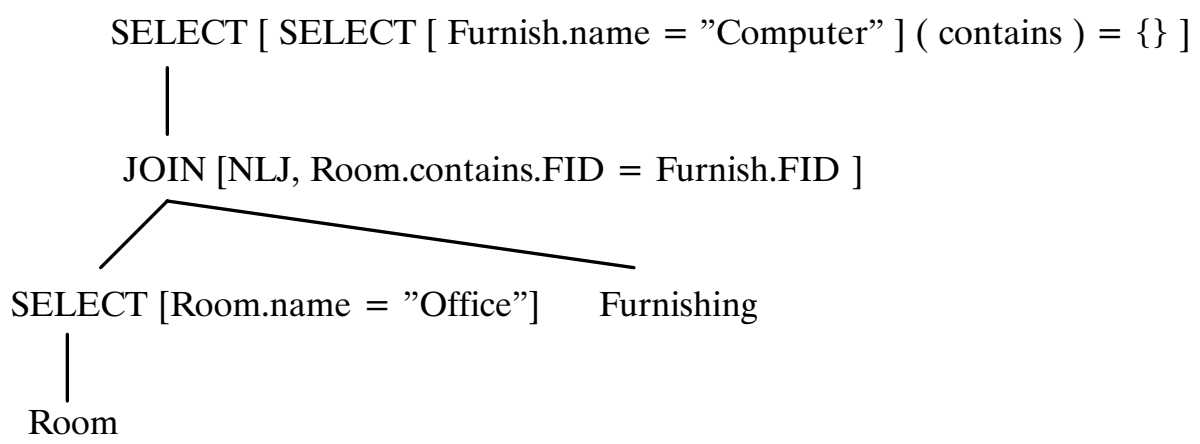


Obviously it improves performance, to split the selection and move one part into the join, that is, to apply the name function first to Room objects, before following the set of links (Room.contains) to Furnishings, to check whether there is no Computer in that Room. Assuming an index on name of rooms, and physical links, this will be quite efficient, much more efficient, than in a flat relational approach. There the Room-Furnishing relationship is modeled by taking the primary key of Rooms into Furnishings (as a foreign key). However, if the physical design is the one given in Figure 3.2, query $\mathrm{Q}_{3}$ results in

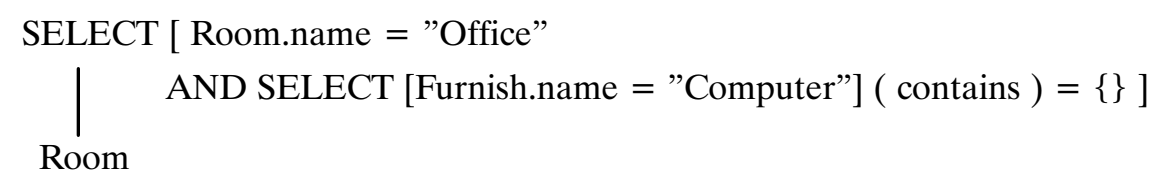

This execution plan does not even need any join operation, and can be executed within one (single scan) kernel operation.

\subsection{Experience}

A first prototype optimizer has been implemented [15, 9], and integrated [41, 19] into the COCOON-DASDBS system. COOL queries are passed from the COOL interface to the optimizer, and after translation onto the physical schema and optimization, execution plans can be passed to the query processor to execute the query on the DASDBS kernel. The query processors functionality includes Nested Loop-, Nested Block-, and Sort Merge Joins, as well as pointerbased variants of these join algorithms, which had been implemented similar to [36]. However notice, all join algorithms perform on nested storage structures, and are therefore variants of the algorithms as known from the relational model. The functionality of the query optimizer includes removal of redundant operations, the combination of operations, and select-project-join ordering, on nested relations. Since our goal was to evaluate the advantages and cost of using a complex record (DASDBS) instead of flat record (RDBMS) storage manager, the main emphasis has been on the effects of hierarchical clustering and embedded references; indexes played only a supporting role; future improvements should include indexes as well.

The implementation of COOL on top of DASDBS has been completed (to the described level) only recently. After evaluating single components, first experiments have been carried out using the entire system. In the following, we will summarize some of the most interesting preliminary results. All experiments were run on a Sun 3/80, without other users.

In order to evaluate the advantages of embedded (sets of) references and hierarchical clustering, we ran the following experiment: We consider a COOL query that accesses two classes, Rooms and Furnishings, and compare object reassembly times, if 1) no physical pointers, 2) physical pointers, and 3) materialization can be exploited. The cardinality of class Room is fixed to 100 tuples, so as to model a selection that has already been evaluated. The number of furnishings per Room, that is, the number of subtuples in the subrelation Room.contains is drawn from a uniform distribution over the range of a) $1-5$, b) 5-10, c) $10-50$ and d) 50-100. This results in a number 
of 300 to 7500 subtuples in total. We evaluated Nested Loop-, Nested Block-, and Sort Merge Joins, as well as their pointer-based variants.

Figure 4.5 compares the best join strategy for either of the three alternatives of physical support (plain value-based, with pointers, materialization). As expected, Sort Merge join performs much better than Nested Loops join, for medium to large relation sizes, both, with and without pointers. We see that storing (sets of) physical object references within the referencing objects, substantially speeds up object reassembly (a factor of 2-3 was observed). This is because the referenced tuples of the second relation can be fetched directly, without scans, index lookups, or sorting. Finally, materializing object functions, as expected, performs best.

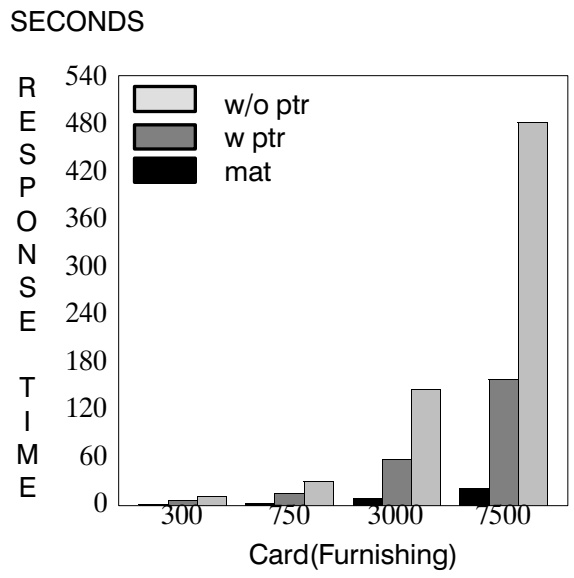

Figure 4.5: Comparrison of Logical References, Physical References and Materialization

The cost function is a linear function of the relation cardinality, compared to e.g. a logarithmic function for Sort Merge join. The improvement increases with the relation cardinality, and ranged in our experiment from a factor 5 to 7 over pointers and 10 to 20 over value-based joins (see also [40]).

We also investigated how much effort had to be spent during the optimization phase. First, we consider the number of nodes created in the search tree for a sample query, relative to the number of joins, as the number of nodes mainly determines the search time. In Figure 4.6, we see that the number of nodes does not increase exponentially, the search time was less than a tenth of a second for each query. Second, we see in Figure 4.7 the estimated execution cost before and after the transformations had been applied, as delivered by EXODUS.

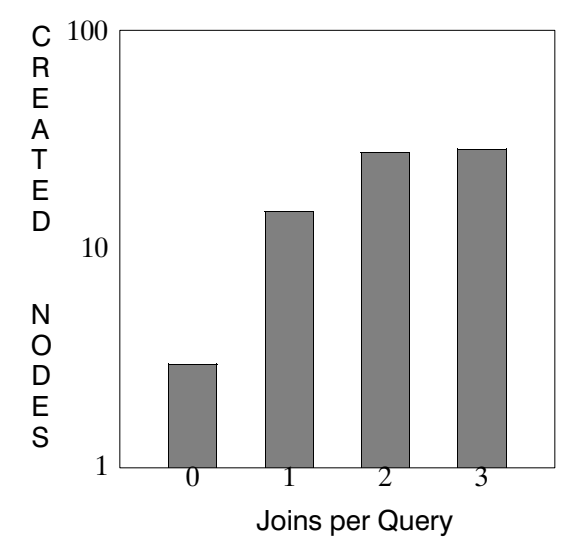

Figure 4.6: MASH Size

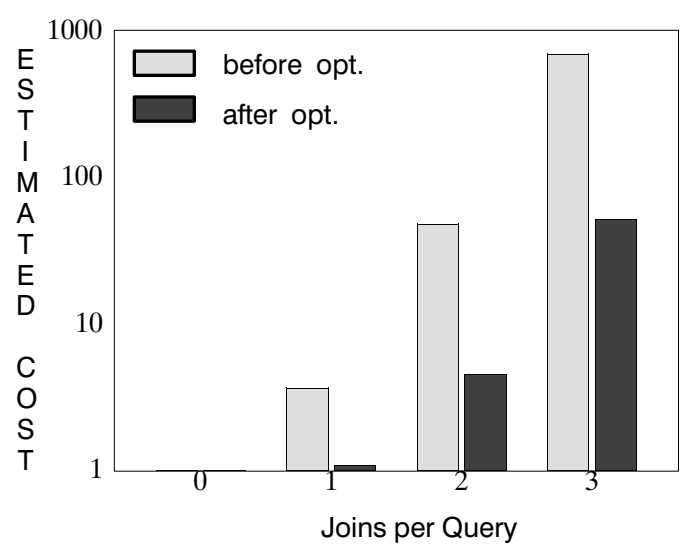

Figure 4.7: Plan Cost before and after Transformations

Our optimization approach, using rule based optimization techniques, showed to handle the optimization task, generating efficient execution plans in a reasonable time. Due to the exploitation 
of hierarchical clustering and embedded (sets of) object references this result in substantial execution time improvements.

The use of DASDBS storage structures (nested relations) and corresponding query processing strategies required a substantial amount of coding (for "argument transfer functions") in addition to the generated optimizer. This is because the EXODUS rule language was oriented towards a flat algebra.

We plan to include more sophisticated query evaluation plans into the optimizers repertoire, for example hierarchicalized join plans [25]. This will, however, require even more hand coding. So, EXODUS might no longer be the tool best suited for this purpose.

The next step is to compare the relative performance of the DASDBS implementation with an implementation based on a commercial platform: Oracle, as a relational system that can emulate two-level nesting (via Oracle "Clusters"). The details of these experiments are beyond the scope of this paper. In a nutshell, the results we obtained so far are: as expected, with the same query processing strategy Oracle performs up to a few times faster than DASDBS. However, one- tomany joins supported by hierarchical clustering in DASDBS outperforms Oracle clusters. Further, many-to-many relationships supported by hierarchical clustering of sets of object references in DASDBS (resulting in just one join operation for reassembling object structures) outperforms Oracle (where two joins are needed, one of them can be supported by a cluster) as well.

\section{Related Work}

Much work in query optimization for OODBMS, has concentrated on optimizing path traversals. Index structures tailored for accessing objects along a reference chain, leading from one object to another via single valued or set valued attributes are considered in e.g. [18, 11, 12, 4, 1, 13, 35]. These proposed strategies are important pieces to enhance performance for path expressions, using supporting index structures. However, our approach does not focus on index structures for path traversals. Rather, we specified a query optimization methodology for exploring object references stored inside the owner object, as well as hierarchical clustering, corresponding to the direct storage model. In addition, our approach tries to exploit set-oriented processing strategies, whereas others, e.g. Kemper's term representation for queries [12] is typically oriented towards nested iteration schemes.

Query rewrite approaches, like [34, 37, 5, 23] are more general then focusing on path traversals, but study query optimization just on the logical level. Choices for physical object representation as well as method implementations are not considered. This precludes the specification of cost based optimization strategies. We consider a variety of physical designs and the resulting costs for complex object processing [40], and are therefore able to estimate the effect of each transformation, based on a cost model. This is similar to [16], which focussed on recursive queries.

In the COCOON project, we study a new query optimization approach, that incorporates features not found in earlier approaches. No other approach we are aware of, has analogs to our uni- 
fied treatment of handling (set valued) object relationships, which may be supported by logical references, physical references or materialization. As queries involving path expressions are very common in object oriented databases, it is important to recognize that certain path traversals can be expressed as joins. These have several possible execution orders, and several possible implementation strategies, depending on the physical database layout. The choice of an efficient strategy is performed by an optimizer constructed using the EXODUS optimizer generator [15]. Notice that dealing with path traversals as joins is related to the transformation of nested SQL queries [14].

\section{Conclusions}

We described the mapping of COCOON to DASDBS, a nested relational database kernel system, as an example OODBMS mapping to a complex storage system. We described 1) choices for physical designs that make use of the complex storage model and 2) the generation of efficient, set-oriented execution plans for object-oriented database queries, using rule-based query optimization techniques.

We use hierarchical clustering and embedded (sets of) object references, as features supported by DASDBS. Therefore, we are able to avoid breaking the complex structures of the objects into small pieces in order to store them in (flat) relations, as this would result in large join queries, for queries against the object-oriented schema. Further, storing (sets of) physical object references, allows efficient pointer-based join algorithms. This results in being superior in performance, due to more flexible physical database layouts and more efficient query processing algorithms. A query optimization approach is described, which handles the required optimization task.

A first physical design tool and a first query optimizer have been implemented. Preliminary results show feasibility, and execution time improvements of an order of magnitude. In the future, we will -among others - compare our approach to a mapping onto a commercial flat record (RDBMS) storage manager.

\section{References}

1. O. Deux et al., "The O2 System", $C A C M$, vol. 34, no. 10, p. 34, October 1991.

2. C. Beeri, "Formal Models for Object Oriented Databases", Proc. DOOD, Kyoto, 1989.

3. E. Bertino, "Optimization of Queries using Nested Indices", in Proc. EDBT, LNCS, vol. 416, pp. 44-59, Springer 1990.

4. E. Bertino and W. Kim, "Indexing Techniques for Queries on Nested Objects", IEEE Trans. on Knowledge and Data Eng., vol. 1, no. 2, p. 196, June 1989.

5. S. Cluet and C. Delobel, "A General Framework for the Optimization of Object-Oriented Queries", Proc. SIGMOD, p. 383, San Diego, California., June 1992. 
6. S. Dessloch, T. Härder, F.-J. Leick, N.M. Mattos, C. Laasch, C. Rich, M.H. Scholl, and H.-J. Schek, "COCOON and KRISYS: A Comparison", in Objektbanken für Experten, ed. R. Bayer, T. Härder, and P.C. Lockemann, Informatik Aktuell, Springer Verlag, 1992.

7. G. Graefe, "Volcano, An Extensible and Parallel Dataflow Query Processing System", July 1990, to appear in IEEE Trans. Knowledge and Data Engineering.

8. G. Graefe and D.J. DeWitt, "The EXODUS Optimizer Generator", Proc. ACM SIGMOD, pp. 160-171, San Francisco, CA., May 1987.

9. J.Hofmann, "Evaluierung eines COOL-Anfrageoptimierers", Semesterarbeit, ETH Zurich, Departement Informatik, Zurich, 1992.

10. A. Kemper, C. Kilger, and G. Moerkotte, "Function Materialization in Object Bases", Proc. ACM SIGMOD, p. 258, Denver, CO, May 1991.

11. A. Kemper and G. Moerkotte, "Advanced Query Processing in Object Bases Using Access Support Relations", Proc. VLDB, p. 290, Brisbane, Australia, 1990.

12. A. Kemper and G. Moerkotte, "Access Support in Object Bases", Proc. ACM SIGMOD, p. 364, Atlantic City, NJ, May 1990.

13. U. Kessler and P. Dadam, "Auswertung komplexer Anfragen an hierarchisch strukturierte Objekte mittels Pfadindexen", Proc. BTW, Kaiserslautern, Germany, March 1991.

14. W. Kim, "On Optimizing an SQL-like Nested Query", $A C M T O D S$, vol. 7, no. 3, September 1982.

15. T. Laes, "Generierung und Evaluierung eines Anfrageoptimierers", Diplomarbeit, ETH Zurich, Departement Informatik, Zurich, 1991.

16. R. Lanzelotte, P. Valduriez, and M. Zait, "Optimization of Object-Oriented Recursive Queries Using Cost-Controlled Strategies”, Proc. ACM SIGMOD, San Diego, California., June 1992.

17. R. Lanzelotte, P. Valduriez, M. Ziane, and J.J. Cheiney, "Optimization of Nonrecursive Queries in OODBs", Proc. DOOD-2, Munich, Germany, December 1991.

18. D. Maier and J. Stein, "Indexing in an Object-Oriented DBMS",Proc. Int'l Workshop on Object-Oriented Database Systems, p. 171, Pacific Grove, CA, September 1986.

19. O. Mayer, "Abbildung und Integration von COOL-DDL auf NF2", Semesterarbeit, ETH Zurich, Departement Informatik, Zurich, 1992.

20. K. Ono and G.M. Lohman, "Extensible Enumeration of Feasible Joins for Relational Query Optimization”, IBM Research Report, RJ 6625 (63936), San Jose, CA, December 1988.

21. G. Pathak and J.A. Blakeley, "Query Optimization in Object-Oriented Databases", Proc. Workshop on Database Query Optimization, OGC, CS Technical Report 89-005, Beaverton, OR., May 1989.

22. H.B. Paul, H.J. Schek, M.H. Scholl, G. Weikum, and U. Deppisch, "Architecture and Implementation of the Darmstadt Database Kernel System", Proc. ACM SIGMOD, p. 196, San Francisco, CA, May 1987.

23. H. Pirahesh, W. Hasan, and J. Hellerstein, "Extensible/Rule Based Query Rewrite Optimization in Starburst", Proc. ACM SIGMOD, p. 39, San Diego, California., June 1992. 
24. C. Rich, "Query Rewrite and Plan Optimization in COCOON, using Hierarchical Algebra Transformations", University of Ulm, Dept. of Computer Science, Technical Report, 1992 (in Preparation).

25. A. Rosenthal, C. Rich, and M.H. Scholl, "Reducing Duplicate Work in Relational Join(s): A Unified Approach", Submitted for publication, also Technical Report NR:172, ETH Zurich, Dept. of Computer Science, 1992.

26. H.J. Schek, H.B. Paul, M.H. Scholl, and G. Weikum, "The DASDBS Project: Objectives, Experiences, and Future Prospects", IEEE Trans. on Knowledge and Data Eng., vol. 2, no. 1, March 1990.

27. M.H. Scholl, H.B. Paul, and H.J. Schek, "Supporting Flat Relations by a Nested Relational Kernel", Proc. VLDB, p. 137, Brighton, England, August 1987.

28. M.H. Scholl, "The Nested Relational Model - - - Efficient Support for a Relational Database Interface", Ph.D. Thesis, Dept. of Computer Science, TU Darmstadt, 1988 . (in German)

29. M.H. Scholl, "Theoretical Foundation of Algebraic Optimization Utilizing Unnormalized Relations", in Proc. ICDT, LNCS, vol. 243, pp. 380-396, Springer, Rome, Italy, September 1986.

30. M.H. Scholl, "Physical Database Design for an Object Oriented Database System", in Query Processing for Advanced Database Applications, ed. J.C. Freytag, G. Vossen, and D. Maier, Morgan-Kaufman, San Mateo, CA, 1993, To appear.

31. M.H. Scholl and H.-J. Schek, "A Relational Object Model", in Proc. ICDT, LNCS, vol. 470, pp. 89-105, Springer, Paris, December 1990.

32. M.H. Scholl and H.-J. Schek, "A Synthesis of Complex Objects and Object-Orientation", Proc. IFIP TC 2 Working Conference on Database Semantics: Object Oriented Databases Analysis, Design and Construction, Windermere, U.K., July 1990.

33. P.G. Selinger, M.M. Astrahan, D.D. Chamberlin, R.A. Lorie, and T.G. Price, "Access Path Selection in a Relational Database Management System", Proc. ACM SIGMOD, pp. 23-34, Boston, MA., May-June 1979.

34. G.M. Shaw and S.B. Zdonik, "A Query Algebra for Object-Oriented Databases", Proc. IEEE Data Eng., p. 154, Los Angelos, CA, February 1990.

35. E. Shekita and M. Carey, "Performance Enhancement Through Replication in an ObjectOriented DBMS", Proc. ACM SIGMOD, p. 325, Portland, OR, May-June 1989.

36. E.J. Shekita and M.J. Carey, "A Performance Evaluation of Pointer-Based Joins", Proc. ACM SIGMOD, p. 300, Atlantic City, NJ., May 1990.

37. D.D. Straube, "Queries and Query Processing in Object-Oriented Database Systems", University of Alberta, CSD Technical Report 90-33, Edmonton, Canada, December 1990.

38. A. Swami, "Optimization of Large Join Queries: Combining Heuristics and Combinatorial Techniques", Proc. ACM SIGMOD, p. 367, Portland, OR, May-June 1989.

39. A. Swami and A. Gupta, "Optimizing Large Join Queries", Proc. ACM SIGMOD, pp. 8-17, Chicago, IL., June 1988. 
40. W.B. Teeuw, C. Rich, M.H. Scholl, and H.M. Blanken, "An Evaluation of Physical Disk I/Os for Complex Object Processing", Proc. IEEE Conf. on Data Eng., Vienna, Austria, April 1993, To appear. A more detailed version is available as Technical Report 183, ETH Zurich, Dept. of Computer Science, 1992.

41. D. Wilhelm, "Query-Schnittstelle für DASDBS", Diplomarbeit, ETH Zurich, Departement Informatik, Zurich, 1991. 